\title{
MINIMAL SKEW PRODUCTS
}

BY

S. GLASNER

\begin{abstract}
Let $(\sigma, Z)$ be a metric minimal flow. Let $Y$ be a compact metric space and let $\mathcal{G}$ be a pathwise connected group of homeomorphisms of $Y$. We consider a family of skew product flows on $Z \times Y=X$ and show that when $(\mathcal{G}, Y)$ is minimal most members of this family have the property of being disjoint from every minimal flow which is disjoint from $(\sigma, Z)$. From this and some further results about skew product flows we deduce the existence of a minimal metric flow which is disjoint from every weakly mixing minimal flow but is not PI.
\end{abstract}

0. In this paper we use the term "flow" to describe a compact Hausdorff space $X$ together with a homeomorphism $T$ of $X$ onto itself. Let $\mathcal{E}$ be the family of equicontinuous minimal flows, let $\mathcal{W}$ be the family of weakly mixing minimal flows and let $\mathscr{P} \mathscr{G}$ be the family of Proximal Isometric (PI) flows. For a class of flows $\mathcal{K}$ we let $\mathcal{K}^{\perp}$ be the class of minimal flows which are disjoint from every member of $\mathscr{K}$. It is well known that $\mathcal{E}^{\perp}=\mathscr{W}$ and that $\mathscr{P} \mathcal{G} \subset \mathscr{S}^{\perp}=\varepsilon^{\perp \perp}$. The main result of this paper is that $\mathscr{P} \mathscr{Q} \neq \mathscr{W}^{\perp}$.

In $\S 1$ we construct a family, $\bar{\delta}_{\mathcal{G}}(\sigma)$, of skew product flows on $X=Z \times Y$ where $(\sigma, Z)$ is a metric minimal flow and $\mathcal{G}$ is a pathwise connected subgroup of homeomorphisms of the compact metric space $Y$. Using the methods of [6] we show that when $(\mathcal{G}, Y)$ is minimal most members of $\overline{\mathcal{S}}_{\mathcal{G}}(\sigma)$ are disjoint from every minimal flow which is disjoint from $(\sigma, Z)$.

In $\$ 2$ we show that if in addition $\mathcal{G}$ is abelian then every member of $\bar{\delta}_{\mathcal{G}}(\sigma)$ is an RIC extension of $(\sigma, Z)$. (See following definitions or see [3].) Combining this result with the results of $\S 1$ and the fact that when $(\mathcal{G}, Y)$ is weakly mixing most members of $\overline{\mathcal{S}}_{\mathcal{G}}(\sigma)$ are weakly mixing extensions of $(\sigma, Z)$ [6, Theorem 4], we deduce the existence of a metric minimal flow which is disjoint from every element of $W$ but is not PI.

1. For a compact metric space $Y$ we let $\mathcal{H}(Y)$ be the space of all homeomorphisms of $Y$ with the metric

$$
d(g, h)=\operatorname{Sup}_{y \in Y} d(g(y), h(y))+\operatorname{Sup}_{y \in Y} d\left(g^{-1}(y), h^{-1}(y)\right) .
$$

With this metric $\mathcal{H}(Y)$ is a complete topological group. Let $\mathcal{G}$ be a subgroup of $\mathcal{H}(Y)$ and let $(\sigma, Z)$ be a minimal metric flow (i.e. $\sigma$ is a homeomorphism of the compact metric space $Z$ and $\sigma A \subset A$ for a closed subset $A$ of $Z$ implies $A=\varnothing$ or $A=Z$ ). With every continuous map $z \rightarrow g_{z}$ of $Z$ into $\mathcal{G}$ we associate a homeomor-

Received by the editors July 25, 1979 and, in revised form, November 30, 1979.

AMS (MOS) subject classifications (1970). Primary 54H20.

Key words and phrases. Minimal flow, weakly mixing, disjointness, PI. 
phism $G$ of $X=Z \times Y$ defined by $G(z, y)=\left(z, g_{z}(y)\right)$. Identify $\sigma$ with $\sigma \times e$ where $e$ is the identity map on $Y$, and then for $(z, y) \in X$

$$
G^{-1} \circ \sigma \circ G(z, y)=\left(\sigma z, g_{\sigma^{-1}} g_{z}(y)\right) \text {. }
$$

Let $\bar{\delta}_{\mathcal{G}}(\sigma)$ be the closure of the subset of $\mathcal{H}(X)$ whose elements are the maps of the form $G^{-1} \circ \sigma \circ G$ as above. Notice that every element $T \in \bar{\delta}_{\mathcal{G}}(\sigma)$ has the form $T(z, y)=\left(\sigma z, h_{z}(y)\right)$, where $z \rightarrow h_{z}$ is a continuous map of $Z$ into the closure of $\mathcal{G}$ in $\mathcal{H}(Y)$. We write $\pi_{Z}=\pi$ for the projection map $X \rightarrow Z$ and $\pi_{Y}$ for the projection $X \rightarrow Y$.

With every minimal flow $(T, X)$ we associate a flow $\left(T, 2^{X}\right)$ on the compact space of closed subsets of $X$ equipped with the Hausdorff topology. A minimal subflow of $\left(T, 2^{X}\right)$ is called a quasifactor of $(T, X)$.

The minimal flows $\left(T, X_{1}\right)$ and $\left(T, X_{2}\right)$ are disjoint if the product flow $\left(T, X_{1} \times\right.$ $\left.X_{2}\right)$ is minimal. By [4, Theorem 2.4], the minimal metric flows $\left(T, X_{1}\right)$ and $\left(T, X_{2}\right)$ are not disjoint iff there exist an almost one-to-one extension $\left(T, X_{1}^{\prime}\right)$ of $\left(T, X_{1}\right)$ and a nontrivial quasifactor of $\left(T, X_{2}\right)$ which is a factor of $\left(T, X_{1}^{\prime}\right)$.

A closed subset $A$ of $X$ is called an almost periodic set if $A$ is an element of some quasifactor of $(T, X)$.

1.1 Proposition. Assume $\mathcal{G}$ is pathwise connected and $(\mathcal{G}, Y)$ is minimal. Then there exists a dense $G_{\delta}$ subset $\Re$ of $\overline{\mathcal{S}}_{\mathcal{G}}(\sigma)$ such that every $T \in \Re$ has the following property. Let $A$ be a closed subset of $X=Z \times Y$ with $\pi(A)=Z$. Then there exist a point $z \in Z$ and a sequence $\left\{n_{i}\right\}$ such that $\lim T^{n_{i}} A \supset \pi^{-1}(z)$. (Notice that this implies the minimality of $(T, X)$.)

Proof. We split the proof into five steps.

1. For every $\varepsilon>0$ let

$$
\begin{aligned}
& E_{\varepsilon}=\left\{T \in \bar{\delta}_{\mathcal{G}}(\sigma): \exists l_{1}, \ldots, l_{L} ; \exists z_{1}, \ldots, z_{N} \in Z\right. \text { such that } \\
& \operatorname{diam}\left(\left\{\sigma^{l j} z_{i}\right\}_{i=1}^{N}\right)<\varepsilon \text { for } 1 \leqslant j \leqslant L \text { and } \forall y_{1}, \ldots, y_{N} \in Y \exists j \\
& \text { with } \left.\pi_{Y}\left(\left\{T^{l}\left(z_{i}, y_{i}\right)\right\}_{i=1}^{N}\right) \varepsilon \text {-dense in } Y\right\} \text {. }
\end{aligned}
$$

$E_{\varepsilon}$ is an open subset of $\overline{\mathcal{S}}_{\mathcal{G}}(\sigma)$. Put $\Re=\cap E_{1 / n}$ then it is clear that every $T \in \Re$ has the desired property. Thus it is enough to show that $E_{\varepsilon}$ is dense in $\bar{\delta}_{\mathcal{G}}(\sigma)$ for every $\varepsilon>0$.

2. It is enough to show that $G \circ \sigma \circ G^{-1} \in \bar{E}_{\varepsilon}$ for every $G$. Since $\mathcal{H}(X)$ is a topological group this is the same as showing that $\sigma \in \overline{G^{-1} E_{\varepsilon} G}$. Now

$$
\begin{array}{r}
G^{-1} E_{\varepsilon} G=\left\{T \in \overline{\mathcal{S}}_{\mathcal{G}}(\sigma): \exists l_{1}, \ldots, l_{L} ; z_{1}, \ldots, z_{N} \in Z\right. \text { such that } \\
\operatorname{diam}\left(\left\{\sigma^{l} z_{i}\right\}_{i=1}^{N}\right) \text { for } 1 \leqslant j \leqslant L \text { and } \forall y_{1}, \ldots, y_{N} \in Y \exists j \\
\text { with } \left.\pi_{Y}\left(G\left\{T^{l}\left(z_{i}, g_{z_{i}}^{-1}\left(y_{i}\right)\right)\right\}_{i=1}^{N}\right) \varepsilon \text {-dense in } Y\right\} .
\end{array}
$$


But the latter set contains $E_{\delta}$ where $\delta>0$ is such that $G$ sends $\delta$-dense sets into $\varepsilon$-dense sets. Therefore $\bar{E}_{\delta} \subset \overline{G^{-1} E_{\varepsilon} G}$ and it is enough to show that $\sigma \in \bar{E}_{\varepsilon}$ for every $\varepsilon>0$.

3. Fix $\varepsilon>0$ and let $Y=\cup_{j=1}^{N} V_{j}$ where the $V_{j}$ 's are open sets with $\operatorname{diam}\left(V_{j}\right)<$ $\varepsilon, j=1, \ldots, N$. Since $\left(\mathcal{G}^{N}, Y^{N}\right)$ is minimal there are $g_{i, j} \in \mathcal{G}, i=1, \ldots, N ; j=$ $1, \ldots, L ;$ with

$$
\bigcup_{j=1}^{L} g_{i, j}^{-1}\left(V_{1}\right) \times \cdots \times g_{N, j}^{-1}\left(V_{N}\right)=\underbrace{Y \times \cdots \times Y}_{N} .
$$

Thus for every vector $\left(y_{1}, \ldots, y_{N}\right) \in Y^{N}$ there exists a $j$ with

$$
\left(g_{1, j}\left(y_{1}\right), \ldots, g_{N_{j}}\left(y_{N}\right)\right) \in V_{1} \times \cdots \times V_{N},
$$

i.e. $\left\{g_{i, j}\left(y_{i}\right)\right\}_{i=1}^{N}$ is $\varepsilon$-dense in $Y$.

4. Let $\delta>0$. We construct a continuous map $z \rightarrow g_{z}, Z \rightarrow \mathcal{G}$, define $G \in \mathcal{H}(X)$ by $G(z, y)=\left(z, g_{z}(y)\right)$ and show that for this $G$ we have

(i) $d\left(\sigma, G^{-1} \circ \sigma \circ G\right)<\delta$,

(ii) $G^{-1} \circ \sigma \circ G \in E_{\varepsilon}$,

thereby proving that $\sigma \in \bar{E}_{\boldsymbol{\varepsilon}}$.

Let $M=N \times L$ and put

$$
h_{0}=e, \quad h_{((j-1) N+i) / M}=g_{i, j}^{-1}, \quad i=1, \ldots, N ; j=1, \ldots, L .
$$

Since $\mathcal{G}$ is pathwise connected the map $t \rightarrow h_{t}$ on the finite set

$$
\{((j-1) N+i) / M\}_{i, j} \cup\{0\}
$$

can be extended to a continuous map $t \rightarrow h_{t}$ on $I=[0,1]$ into $\mathcal{G}$. Let $\eta>0$ have the property: $\left|t_{1}-t_{2}\right|<\eta$ implies $d\left(h_{t_{1}}^{-1} h_{t_{2}}, e\right)<\delta$. Choose $n$ with $2 / n<\eta$. Let $W \subset Z$ be an open set such that for some $l_{1}<l_{2}<\cdots<l_{L}$, with $i>j \Rightarrow l_{i}-l_{j}$ $>n$, for all $j, \operatorname{diam}\left\{\sigma^{l_{j}} W\right\}<\varepsilon$ and $\sigma^{l_{j}+s} W \cap \sigma^{l_{i}+t} W=\varnothing$ whenever $(j, s) \neq$ $(i, t), i, j=1, \ldots, L, s, t=0,1, \ldots, n-1$. Let $K \subset W$ be a Cantor set, let $l_{0}=$ 0 and let $\tilde{\theta}: \cup_{j=0}^{L} \sigma^{l_{j}} K \rightarrow I$ be a continuous map such that $\tilde{\theta}(K)=0, \tilde{\theta}$ maps $\sigma^{l_{1}} K$ onto $[0, N / M]$ and for $j>1$ and $z \in \sigma^{l_{j} K}, \tilde{\theta}(z)=\tilde{\theta}\left(\sigma^{l_{1}-l_{j}}\right)+N(j-1) / M$. For $0 \leqslant j \leqslant L, 0 \leqslant i \leqslant n-1$ and $z \in \sigma^{l_{j}+i} K$ let $\tilde{\theta}(z)=\tilde{\theta}\left(\sigma^{-i} z\right)$. Now extend $\tilde{\theta}$ to a continuous map $\tilde{\theta}: Z \rightarrow I$. Put $\theta(z)=\sum_{i=0}^{n-1} \tilde{\theta}\left(\sigma^{i} z\right) / n(z \in Z)$, and define $z \rightarrow g_{z}$; $Z \rightarrow \mathcal{G}$ by $g_{z}=h_{\theta(z)}$. Finally let $G \in \mathcal{H}(X)$ be defined by $G(z, y)=\left(z, g_{z}(y)\right)$ $((z, y) \in X)$.

5. For $(z, y) \in X$,

$$
G^{-1} \circ \sigma \circ G(z, y)=\left(\sigma z, g_{\sigma z}^{-1} g_{z}(y)\right)=\left(\sigma z, h_{\theta(\sigma z)}^{-1} h_{\theta(z)}(y)\right) .
$$

But

$$
\begin{aligned}
|\theta(\sigma z)-\theta(z)| & =\frac{1}{n}\left|\sum_{i=0}^{n-1} \tilde{\theta}\left(\sigma^{i+1} z\right)-\tilde{\theta}\left(\sigma^{i} z\right)\right| \\
& =\frac{1}{n}\left|\tilde{\theta}\left(\sigma^{n} z\right)-\tilde{\theta}(z)\right| \leqslant \frac{2}{n}<\eta .
\end{aligned}
$$

Thus $d\left(h_{\theta(\sigma z)}^{-1} h_{\theta(z)}, e\right)<\delta$ and (i) is proved. 
Let $z_{1}, \ldots, z_{N} \in K$ be chosen so that

$$
\tilde{\theta}\left(\sigma^{l} z_{i}\right)=i / M, \quad i=1,2, \ldots, N .
$$

Then for $1 \leqslant j \leqslant L, \theta\left(\sigma^{l} z_{i}\right)=((j-1) N+i) / M$ and $\theta\left(z_{i}\right)=0, i=1, \ldots, N$. Now given $y_{1}, \ldots, y_{N} \in Y$ there exists a $j, 1 \leqslant j \leqslant L$, with $\left\{g_{i, j}\left(y_{i}\right)\right\}_{i=1}^{N} \varepsilon$-dense in $Y$, thus for that $j$

$$
\begin{aligned}
\pi_{Y}\left\{\left(G^{-1} \circ \sigma \circ G\right)^{l_{j}}\right. & \left.\left(z_{i}, y_{i}\right): i=1, \ldots, N\right\} \\
& =\pi_{Y}\left\{\left(\sigma^{l_{j}} z_{i}, g_{\sigma_{j z_{i}}}^{-1} g_{z_{i}}\left(y_{i}\right)\right): i=1, \ldots, N\right\} \\
& =\left\{g_{\sigma^{j} z_{i}}^{-1}\left(y_{i}\right)\right\}_{i=1}^{N}=\left\{h_{((j-1) N+i) / M}^{-1}\left(y_{i}\right)\right\}_{i=1}^{N} \\
& =\left\{g_{i, j}\left(y_{i}\right)\right\}_{i=1}^{N} .
\end{aligned}
$$

The latter is $\varepsilon$-dense in $Y$ and (ii) is proved. The proof of the proposition is now complete.

1.2 Corollary. For $T \in \mathscr{R},(T, X)$ has no nontrivial quasifactors which are disjoint from $(\sigma, Z)$.

Proof. Let $\mathscr{X} \subset 2^{X}$ be a quasifactor of $(T, X)$ which is disjoint from $(\sigma, Z)$, i.e., $(\sigma \times T, Z \times \mathscr{X})$ is minimal. Then $\pi(\mathscr{X})=\{\pi(A): A \in \mathfrak{X}\} \subset 2^{Z}$ is a quasifactor of $(\sigma, Z)$. Since $\mathcal{X}$ is disjoint from $(\sigma, Z), \pi(X)=\{Z\}$. Thus $\pi(A)=Z$ for every $A \in \mathcal{X}$ and for every pair $(z, A), z \in Z, A \in \mathcal{X}$ the set $A_{z}=\pi^{-1}(z) \cap A$ is nonempty. Clearly the map $(z, A) \rightarrow A_{z}, Z \times \mathscr{X} \rightarrow 2^{X}$ is upper-semicontinuous. Moreover

$$
(\sigma, T)(z, A)=(\sigma z, T A) \rightarrow T A \cap \pi^{-1}(\sigma z)=T\left(A \cap \pi^{-1}(z)\right),
$$

and therefore $(T A)_{\sigma z}=T\left(A_{z}\right)$.

Fix $A \in \mathcal{X}$ for which there exists $z_{0} \in Z$ with $\pi^{-1}\left(z_{0}\right) \subset A$. Let $z \in Z$. Then since $Z \times \mathscr{X}$ is minimal, there exists a sequence $\left\{n_{i}\right\}$ such that

$$
\lim (\sigma, T)^{n_{i}}\left(z_{0}, A\right)=(z, A) .
$$

By upper-semicontinuity

$$
\lim T^{n_{i}}\left(\pi^{-1}\left(z_{0}\right)\right)=\lim T^{n_{i}}\left(A_{z_{0}}\right)=\lim \left(T^{n_{i}} A\right)_{\sigma^{n_{i} z_{0}}} \subset A_{z} .
$$

On the other hand, since $\pi$ is an open map,

$$
\lim T^{n_{i}}\left(\pi^{-1}\left(z_{0}\right)\right)=\pi^{-1}(z) .
$$

Thus $A \supset \pi^{-1}(z)$ for every $z \in Z$ and $A=X$, i.e., $\mathcal{X}$ is trivial.

1.3 TheORem. Let $(\sigma, Z)$ be a metric minimal flow. Let $Y$ be a compact metric space and $\mathcal{G}$ a pathwise connected subgroup of $\mathcal{H}(Y)$, such that $(\mathcal{G}, Y)$ is minimal. Then there exists a dense $G_{\delta}$ subset $\Re$ of $\overline{\mathcal{S}}_{\mathcal{G}}(\sigma)$ such that for every $T \in \Re$ the corresponding flow $(T, X)$ is minimal and disjoint from every minimal flow which is disjoint from $(\sigma, Z)$. 
Proof. Let $\Re$ be as in Proposition 1.1. Then our theorem follows for $T \in \mathscr{R}$ by Corollary 1.2, the fact that disjointness is preserved under almost one-to-one extensions and by [4, Theorem 2.4$]$.

Let $\mathbf{T}=\mathbf{R} / \mathbf{Z}$ be the 1-torus, let $\alpha$ be an irrational number and let $(\sigma, Z)=$ $\left(R_{\alpha}, \mathbf{T}\right)$ where $R_{\alpha} z=z+\alpha$. Define $T: \mathbf{T}^{2} \rightarrow \mathbf{T}^{2}$ by $T(z, y)=(z+\alpha, y+\phi(z))$, where $\phi: \mathbf{T} \rightarrow \mathbf{T}$ is a continuous function. When $\phi(z) \equiv \beta$ for some $\beta \in \mathbf{T},\left(T, \mathbf{T}^{2}\right)$ is a product flow and while $\left(R_{\alpha}, \mathrm{T}\right)$ is disjoint from $\left(R_{\beta}, \mathbf{T}\right)$, for $\beta$ independent of $\alpha$, $\left(T, \mathbf{T}^{2}\right)$ admits $\left(R_{\beta}, \mathbf{T}\right)$ as a factor. However, under the assumption that $\left(T, \mathbf{T}^{2}\right)$ is not an equicontinuous flow (for $\phi$ this means that for every complex number $\lambda$ and integer $k \neq 0$ the functional equation $f(z+\alpha) e^{2 \pi i k \phi(z)}=\lambda f(z)$ has no nonzero continuous solution; in particular this implies that $\left(T, \mathbf{T}^{2}\right)$ is minimal) it is shown in [5, Theorem 4.2] that if $A$ is an almost periodic closed subset of $\mathbf{T}^{2}$ with $\pi(A)$ second category at $z \in \mathbf{T}$ then $\{z\} \times \mathbf{T} \subset A$. As in the proof of Theorem 1.3 we deduce the following theorem.

1.4 TheOREM. Let $\alpha$ be an irrational and let $\phi: \mathbf{T} \rightarrow \mathbf{T}$ be a continuous function. Let $T: \mathbf{T}^{2} \rightarrow \mathbf{T}^{2}$ be defined by $T(z, y)=T(z, y)=(z+\alpha, y+\phi(z))$. If $\left(T, \mathbf{T}^{2}\right)$ is not equicontinuous then the minimal flow $\left(T, \mathbf{T}^{2}\right)$ is disjoint from every minimal flow which is disjoint from $\left(R_{\alpha}, \mathbf{T}\right)$.

2. Let $\mathcal{G}$ be a topological group, we denote by $\beta \mathcal{G}_{d}$ the Stone-Čech compactification of the discrete underlying group $\mathcal{S}_{d}$. If $(\mathcal{G}, X)$ is a $\mathcal{G}$-flow there is an action of $\beta \mathcal{G}_{d}$ on $X$, written $(p, x) \rightarrow p x\left(p \in \beta \mathcal{G}_{d}, x \in X\right)$, which extends the action of $\mathcal{G}$ on $X$. When both $\mathcal{G}$-flows, $(\mathcal{G}, X)$ and $\left(\mathcal{G}, 2^{X}\right)$, are considered we let $(p, A) \rightarrow$ $p \circ A$ denote the action of $\beta \mathcal{G}_{d}$ on $2^{X}$, rather than $(p, A) \rightarrow p A$. The latter will denote the set $p A=\{p x: x \in A\}$.

$\beta \mathcal{G}_{d}$ has a semigroup structure and the minimal left ideals in $\beta \mathcal{G}_{d}$ coincide with the minimal sets of the $\mathcal{G}$-flow $\left(\mathcal{G}, \beta \mathcal{G}_{d}\right)$. All these minimal sets are isomorphic. Fix a minimal ideal $M$ in $\beta \mathcal{G}_{d}$ and let $u$ be an idempotent in $M$. If $\left(\mathcal{G}, X_{1}\right)$ and $\left(\mathcal{G}, X_{2}\right)$ are two minimal $\mathcal{G}$-flows and $X_{1} \stackrel{\pi}{\rightarrow} X_{2}$ is a homomorphism (or an extension), we say that $\pi$ is an RIC-extension if for some $x \in X_{2}$ and every $p \in M, p \circ u \pi^{-1}(x)=$ $\pi^{-1}(p x)$. This definition does not depend on the choice of $M$ or $u$. (For more details we refer the reader to [1], [2], [3].) When $(T, X)$ is a flow the acting group is the group of integers $\mathbf{Z}$.

As in $\S 1$, let $(\sigma, Z)$ be a metric minimal flow, let $Y$ be a compact metric space and $\mathcal{G}$ a subgroup of $\mathcal{H}(Y)$. Let $z \rightarrow g_{z}$ be a continuous map of $Z$ into $\mathcal{G}$ and define a homeomorphism $T$ of $X=Z \times Y$ by $T(z, y)=\left(\sigma z, g_{z}(y)\right)$.

2.1 Proposition. If $(T, X)$ is minimal, $\mathcal{G}$ abelian and $(\mathcal{G}, Y)$ is minimal, then the extension $(T, X) \stackrel{\pi}{\rightarrow}(\sigma, Z)$ is $R I C$.

Proof. Let $M$ be a minimal ideal in $\beta \mathbf{Z}$ and let $u$ be a minimal idempotent in $M$. Let $\left\{n_{i}\right\}$ be a net in $\mathbf{Z}$ converging to $u$. Pick $x_{0}=\left(z_{0}, y_{0}\right) \in X$ with $u x_{0}=x_{0}$; then $u z_{0}=z_{0}$ and $u \pi^{-1}\left(z_{0}\right)=\left\{z_{0}\right\} \times K$ where $K \subset Y$. For each $y \in Y$ we have

$$
u\left(z_{0}, y\right)=\lim T^{n_{i}}\left(z_{0}, y\right)=\lim \left(\sigma^{n_{i}} z_{0}, g_{\sigma^{n_{i}-1_{z}}} g_{\sigma^{n_{i}-z_{2}}} \cdots g_{z_{0}}(y)\right)=\left(x_{0}, y^{\prime}\right)
$$


where $y^{\prime} \in K$. Let $u^{\prime}=\lim g_{\sigma^{n_{i}-1_{z_{0}}}} \circ \cdots \circ g_{z_{0}}$ be an element of $\beta \mathcal{G}_{d}$, then

$$
u \circ u \pi^{-1}\left(z_{0}\right)=u \circ\left(\left\{z_{0}\right\} \times K\right)=\left\{z_{0}\right\} \times\left(u^{\prime} \circ K\right)=\left\{z_{0}\right\} \times\left(u^{\prime} \circ u^{\prime} Y\right) .
$$

Since $\mathcal{G}$ is abelian $u^{\prime} Y$ is dense in $Y$. Hence $u^{\prime} \circ u^{\prime} Y=Y$ and $u \circ u \pi^{-1}\left(z_{0}\right)=$ $\pi^{-1}\left(z_{0}\right)$. If $p \in M$ then, since $\pi$ is an open map, we have

$$
\begin{aligned}
p \circ u \pi^{-1}\left(z_{0}\right) & =p \circ u \circ\left(\left\{z_{0}\right\} \times K\right)=p \circ\left(\left\{z_{0}\right\} \times Y\right) \\
& =p \circ \pi^{-1}\left(z_{0}\right)=\pi^{-1}\left(p z_{0}\right),
\end{aligned}
$$

and $\pi$ is RIC.

2.2 TheOREM. There exists a metric minimal flow which is disjoint from every minimal weakly mixing flow and is not PI.

Proof. Let $(\sigma, Z)$ be an equicontinuous metric minimal flow. Let $(R, Y)$ be a weakly mixing metric minimal real-flow. We let $R \subset \bar{\delta}_{\mathbf{R}}(\sigma)$ be as in Proposition 1.1. By [6, Theorems 1 and 4] there is a dense $G_{\delta}$ subset $\mathcal{R}^{\prime}$ of $\bar{\delta}_{\mathbf{R}}(\sigma)$ which consists of elements $T$ for which $(T, X)$ is a weakly mixing extension of $(\sigma, Z)$. Let $T \in R \cap \Re^{\prime}$. Then $(T, X)$ is a minimal flow, the extension $(T, X) \stackrel{\pi}{\rightarrow}(\sigma, Z)$ is weakly mixing and, by Theorem 1.3 , every minimal flow which is disjoint from $(\sigma, Z)$ is also disjoint from $(T, X)$. In particular $(T, X)$ is disjoint from every weakly mixing minimal flow.

Let $\overline{\mathbf{R}}$ be the uniform closure of $\mathbf{R}$ in $\mathcal{H}(Y)$, then $\overline{\mathbf{R}}$ is abelian. As was remarked in $\S 1$, every element of $\overline{\mathcal{S}}_{\mathbf{R}}(\sigma)$, and in particular $T$, has the form $T(z, y)=$ $\left(\sigma z, g_{z}(y)\right)$ for some continuous map $z \rightarrow g_{z}$ of $Z$ into $\overline{\mathbf{R}}$. Thus Proposition 2.1 applies and the extension $(T, X) \stackrel{\pi}{\rightarrow}(\sigma, Z)$ is RIC.

Now $(\sigma, Z)$ is the maximal equicontinuous factor of $(T, X)$, i.e., $(\sigma, Z)$ is the first step of the canonical PI-tower of $(T, X)$. Since $(T, X) \stackrel{\pi}{\rightarrow}(\sigma, Y)$ is RIC and weakly mixing, $(\sigma, Z)$ is also the last step [3, Theorem X.2.1.]. Thus in the notations of [3], $X=X_{\infty} \neq Y_{\infty}=Z$ and by [3, Theorem X.4.2.], $X$ is not PI.

\section{REFERENCES}

1. R. Ellis, Lectures on topological dynamics, Benjamin, New York, 1969.

2. R. Ellis, S. Glasner and L. Shapiro, PI-flows, Advances in Math. 97 (1975), 148-171.

3. S. Glasner, Proximal flows, Lecture Notes in Math., vol. 517, Springer-Verlag, Berlin and New York, 1976.

4. __ Compressibility properties in topological dynamics, Amer. J. Math. 97 (1975), 148-171.

5. __ Almost periodic sets and measures on the torus, Israel J. Math. 32 (1979), 161-172.

6. S. Glasner and B. Weiss, On the construction of minimal skew products, Israel J. Math. 34 (1979), 321-336.

Department of Mathematics, Tel-Aviv University, Tel-Aviv, IsRael 